\title{
Effect of N-Methyl-N-Nitrosourea on Helicobacter-induced Gastric Carcinogenesis in C57BL/6 Mice
}

\section{Ju Yup Lee ${ }^{1,3}$, Nayoung Kim ${ }^{1}$, Yoon Jeong Choi ${ }^{1}$, Ryoung Hee Nam¹, Yoon Jin Choi', Seonmin Lee', Daeun Choi', Hye Seung Lee ${ }^{2}$, Jin-Wook Kim ${ }^{1}$, Dong Ho Lee ${ }^{1}$}

Departments of ${ }^{1}$ Internal Medicine and ${ }^{2}$ Pathology, Seoul National University Bundang Hospital, Seongnam, ${ }^{3}$ Department of Internal Medicine, Keimyung University School of Medicine, Daegu, Korea

\begin{abstract}
Background: The aim of this study was to investigate the effect of $\mathrm{N}$-methyl-N-nitrosourea (MNU) treatment followed by chronic Helicobacter pylori SS1 and $\mathrm{H}$. felis colonization on the stomachs of C57BL/6 mice. The role of MNU and Helicobacter species in gastric carcinogenesis was also elucidated.

Methods: A total of 69 C57BL/6 mice at 4 weeks of age were divided into 6 groups according to MNU treatment and $H$. pylori SS1 or $\mathrm{H}$. felis infection. The mice were sacrificed at 21 and 50 weeks. The degree of inflammation was determined by histopathology. The levels of gastric mucosal myeloperoxidase, TNF- $\alpha$, and interleukin-1 $\beta$ (IL-1 $\beta$ ) were measured by ELISA.

Results: In the $H$. felis groups with or without MNU, the incidence of gastric tumors was $21.1 \%$ and $35.0 \%$ at 21 and 50 weeks, respectively. No gastric tumors were observed in all control mice. At 50 weeks, 37.5\% of gastric adenoma cases were observed in the $H$. felis alone and MNU $+\mathrm{H}$. felis groups. Furthermore, $12.5 \%$ of gastric adenocarcinoma cases were observed in the MNU alone and MNU $+H$. felis groups. The gastric mucosal IL-1 $\beta$ level was significantly higher in the MNU $+H$. felis group at 21 weeks and $H$. felis group at 50 weeks, respectively, than that for control mice $(P<0.05)$. However, the effect of MNU on $H$. pylori SS1-induced gastric carcinogenesis was low compared to that on $\mathrm{H}$. felis.

Conclusions: Administration of MNU before $H$. felis infection provokes severe inflammation through IL-1 $\beta$, and eventually induces gastric cancer. However, the role of MNU in $H$. pylori SS1-induced gastric carcinogenesis model is minor.
\end{abstract}

(J Cancer Prev 2016;21:182-186)

Key Words: Helicobacter felis, Helicobacter pylori, Methylnitrosourea, Gastric neoplasm

\section{INTRODUCTION}

Gastric cancer is one of the major causes of cancer-related deaths in Korea and other Asian countries. Animal models play an important role in understanding gastric carcinogenesis. After the discovery of Helicobacter pylori, animal models of Helicobacter-associated gastric carcinogenesis have been an important area research. ${ }^{1,2}$ Other etiological factors for gastric cancer, such as salt, nitrates/nitrites, and nitrosamines are known to induce gastric cancer. Among these are the synthetic $\mathrm{N}$-nitroso compounds, such as N-methyl-N-nitrosourea (MNU), which has been widely used in experimental gastric carcinogenesis. ${ }^{3} \mathrm{MNU}$ is an alkylating agent that can potentially induce the formation of DNA adducts and GC $\rightarrow$ TA transition mutations, ${ }^{4}$ and is also known to modify amino acids in histone proteins leading to chromatin remodeling. ${ }^{5}$

While a chronic colonization of the mouse stomach by $H$. felis and $H$. pylori SS1 leads to chronic gastritis and premalignant lesions, there is an important difference between the two. Almost all $H$. felis infections in the C57BL/6 mice develop into gastric adenocarcinoma via sequential steps from intestinal metaplasia and dysplasia, ${ }^{6,7}$ which is very similar to human gastric car-

Received September 7, 2016, Revised September 11, 2016, Accepted September 13, 2016

Correspondence to: Nayoung Kim

Department of Internal Medicine, Seoul National University Bundang Hospital, 82 Gumi-ro 173beon-gil, Bundang-gu, Seongnam 13620, Korea Tel: +82-31-787-7008, Fax: +82-31-787-4051, E-mail: nayoungkim49@empas.com, ORCID: Nayoung Kim, http://orcid.org/0000-0002-9397-0406

Copyright (C) 2016 Korean Society of Cancer Prevention

(c) This is an Open Access article distributed under the terms of the Creative Commons Attribution Non-Commercial License (http://creativecommons.org/licenses/by-nc/4.0) which permits unrestricted non-commercial use, distribution, and reproduction in any medium, provided the original work is properly cited. 
cinogenesis. In contrast, $H$. pylori SS1 failed to induce gastric adenocarcinoma in the majority of mouse species. ${ }^{8}$ We previously demonstrated that inflammation induced by $H$. felis infection was more severe than that from infection with the $H$. pylori SS1 strain, and it finally led to adenocarcinoma in C57BL/6 mice models. ${ }^{9}$ Several animal studies demonstrated that the combination of MNU and Helicobacter spp. infection led to rapid induction of gastric cancer ${ }^{10,11}$ and to a certain extent mimics the proposed pathogenesis of human carcinogenesis. ${ }^{8}$

The aim of this study was to investigate the effect of MNU treatment followed by chronic $H$. pylori SS1 and H. felis colonization on the stomachs of C57BL/ 6 mice. The role of MNU and Helicobacter spp. in inflammatory reactions of gastric carcinogenesis, including the production of pro-inflammatory cytokines, was also elucidated.

\section{MATERIALS AND METHODS}

\section{Animals}

Four-week-old male C57BL/6 mice (Orient Co., Ltd., Seoul, Korea) were used for the experiments. All mice were housed in cages maintained at $23^{\circ} \mathrm{C}$ with a $12 / 12$-hour light/dark cycle under specific pathogen-free conditions. All experimental procedures were approved by the Institutional Animal Care and Use Committee (IACUC) of Seoul National University Bundang Hospital (BA1401-144/001-02).

\section{Experimental design}

Mice were divided into the following six groups: Group 1, control; Group 2, MNU alone; Group 3, H. pyloriSS1 alone; Group 4, H. felis alone; Group 5, MNU + H. pyloriSS1; Group 6, MNU + H. felis. MNU (Sigma Chemical Co., St. Louis, MO, USA) was dissolved in distilled water at a concentration of 200 ppm and administered in drinking water in light-shielded bottles ad libitum. Mice in the MNU groups (Groups 2, 5, and 6) were given drinking water containing 200 ppm MNU, biweekly, for a total of 10 weeks. After completion of MNU administration, mice in the Helicobacter species alone groups (Groups 3 and 4) and MNU + Helicobacter species group (Groups 5 and 6) were inoculated orogastrically with $1 \times 10^{10}$ colony-forming units/mL of $H$. pylori SS1 and $H$. felis (ATCC 49179), five times every alternate day (Fig. 1). The mice were sacrificed by $\mathrm{CO}_{2}$ asphyxiation at 21 and 50 weeks after inoculation.

\section{Histopathology}

At necropsy, stomach tissue was taken from the greater curvature beginning at the squamocolumnar junction and ending at the gastroduodenal junction. Linear gastric strips were fixed in $10 \%$ formalin solution, processed by standard methods, embedded in paraffin, sectioned at $5 \mu \mathrm{m}$, and stained with H\&E. The stomach mucosa was histologically examined for inflammatory and epithelial changes, and for the presence of $H$. pylori or $H$. felis. The degree of neutrophil infiltration, mononuclear cell infiltration, atrophy, and metaplasia was assessed according to

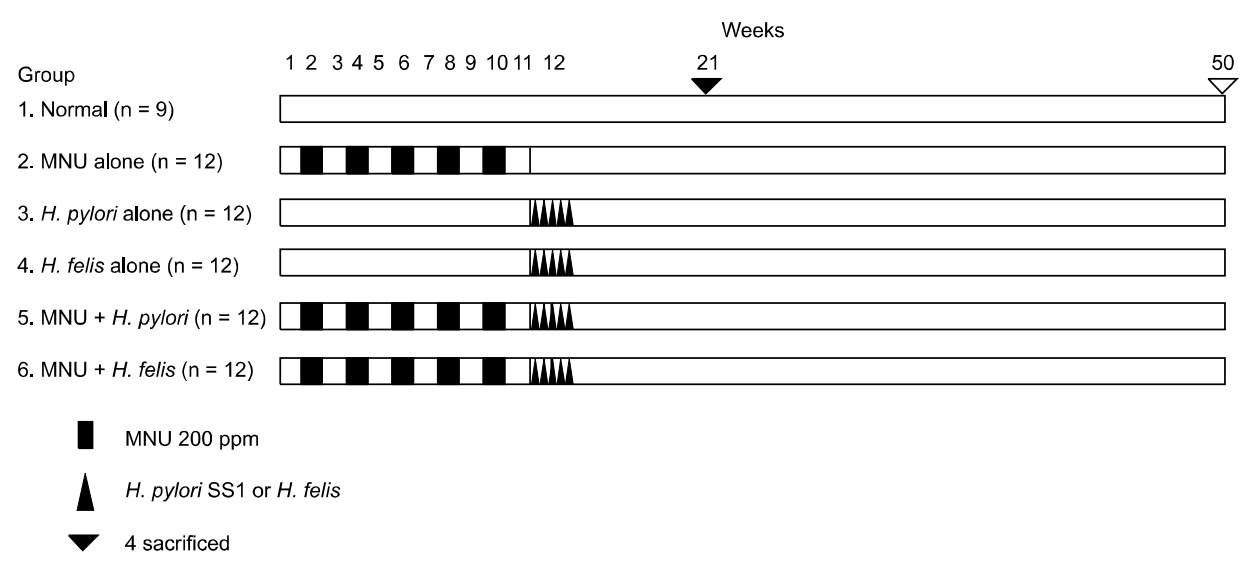

Figure 1. Protocol of the study. Mice were divided into the six groups: Group 1, control; Group 2, N-methyl-N-nitrosourea (MNU) alone; Group 3, Helicobacter pylori SS1 alone; Group 4, H. felis alone; Group 5, MNU + H. pylori SS1; Group 6, MNU + H. felis. MNU was dissolved in distilled water at a concentration of $200 \mathrm{ppm}$ and administered in drinking water in light-shielded bottles ad libitum biweekly, for a total of 10 weeks. After completion of MNU administration, mice in the Helicobacter species alone groups (Groups 3 and 4) and $\mathrm{MNU}+$ Helicobacter species group (Groups 5 and 6 ) were inoculated orogastrically with $1 \times 10^{10}$ colony-forming units/mL of $H$. pylori SS1 and H. felis (ATCC 49179), five times every alternate day. The mice were sacrificed by $\mathrm{CO}_{2}$ asphyxiation at 21 and 50 weeks after inoculation. 
the updated Sydney classification as follows: 0 , absent; 1 , minimal; 2, mild; 3, moderate; 4, marked.

\section{Measurement of mucosal myeloperoxidase, TNF- $\alpha$, and interleukin-1 $\beta$}

Scraped mucosa (10 mg) was homogenized for 30 seconds with a Polytron homogenizer in $200 \mu \mathrm{L}$ of ice-cold lysis buffer $(200 \mathrm{mM}$ $\mathrm{NaCl}, 5 \mathrm{mM}$ EDTA, $10 \mathrm{mM}$ Tris [pH 7.4], 10\% glycerin, $1 \mathrm{mM}$ phenylmethanesulfonyl fluoride, $1 \mu \mathrm{g} / \mathrm{mL}$ leupeptin, and 28 $\mu \mathrm{g} / \mathrm{mL}$ aprotinin). The cell suspensions were centrifuged at 13,000 rpm for 15 minutes and the resulting supernatant was assayed using a myeloperoxidase (MPO) ELISA kit (HyCult Biotechnology, Uden, The Netherlands). For TNF- $\alpha$ and interleukin- $1 \beta$ (IL-1 $\beta$ ), the appropriate kits from R\&D Systems (Minneapolis, MN, USA) were used following the manufacturer's instructions. Protein concentration was measured using a Bio-Rad Protein Assay Kit (Bio-Rad Laboratories, Hercules, MA, USA). The concentration of each cytokine was measured in picograms per milligram of protein. All assays were performed in triplicate.

\section{Statistical analysis}

Data are expressed as the mean \pm SEM. Comparison between the two groups (experimental and control) was performed using the Mann-Whitney U-test. $P<0.05$ was considered to indicate a statistically significant result. All statistical analyses were performed using IBM SPSS software ver. 20.0 (IBM Co., Armonk, NY, USA).

\section{RESULTS}

\section{Gastric tumor incidence and histopathology}

At 21 weeks, among 19 experimental mice, 4 mice (1 hyperplastic polyp and 1 adenoma in the $H$. felis alone group; 2 hyperplastic polyps in the $\mathrm{MNU}+H$. felis group) had gastric tumors and the incidence of gastric tumors was $21.1 \%$. At 50 weeks, among 40 experimental mice, 14 mice had gastric tumors and the incidence of gastric tumors was $35.0 \%$. In the experimental group, $37.5 \%$ of gastric adenomas were observed in the $H$. felis and MNU $+H$. felis groups, respectively, and $25.0 \%$ of gastric adenomas were identified in the $H$. pylori group. Furthermore, $12.5 \%$ of gastric adenocarcinomas were observed in the MNU alone and the $\mathrm{MNU}+\mathrm{H}$. felis group, respectively. In contrast, no gastric tumors were observed in all control mice (Fig. 2A). The neutrophil and monocyte grades of Groups 2, 4, 5, and 6 were increased compared to those of the control group at 21 weeks and the neutrophil and monocyte grades of Groups 3, 4, and 6 were increased compared to those of the control group at 50 weeks (Fig. 2B).

\section{Expression of pro-inflammatory cytokines}

At 21 weeks, the gastric mucosal MPOlevel in $H$. felis-infected mice was significantly higher $(P<0.05)$ than that in control mice (Fig. 3A). At 50 weeks, the gastric mucosal MPO level in the MNU $+H$. pylorigroup was significantly higher $(P<0.05)$ than that in control mice (Fig. 3A). The gastric mucosal IL-1 $\beta$ level was significantly higher for the $\mathrm{MNU}+H$. felis group at 21 weeks and the $H$. felis group at 50 weeks, respectively, than that for control

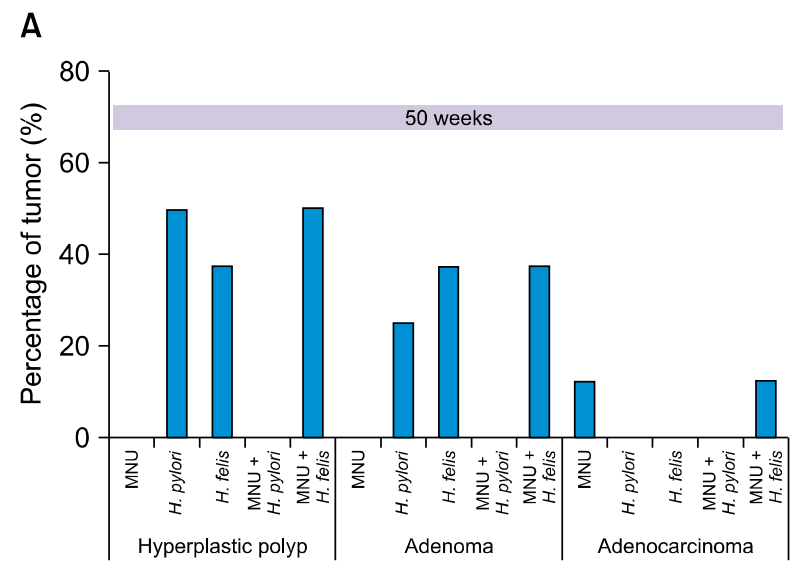

B

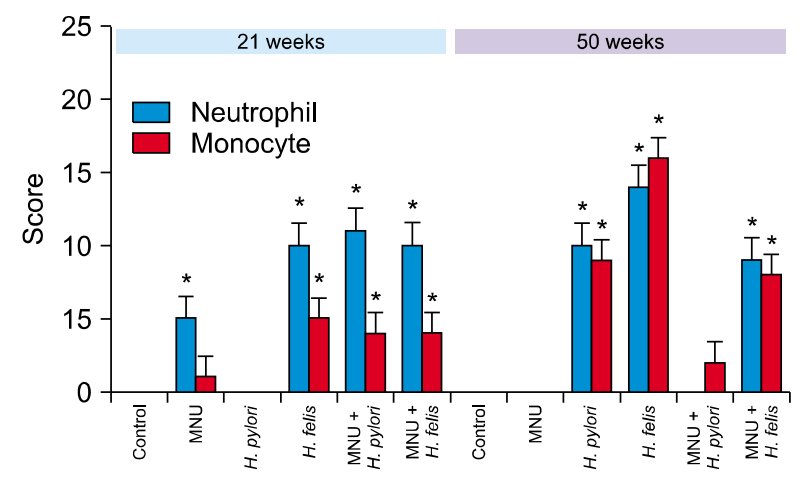

Figure 2. The incidence of gastric hyperplastic polyps, adenoma, and adenocarcinoma at 50 weeks and histologic findings at 21 and 50 weeks. Stomach tissue was taken from the greater curvature beginning at the squamocolumnar junction and ending at the gastroduodenal junction. (A) Hyperplastic polyp, adenoma, and adenocarcinoma were detected using a stereomicroscope and histopathological examination. The incidence was calculated as percentage of tumor-bearing mice/total mice in each experimental group. (B) The degree of histological neutrophil and mononuclear cell infiltration (0, absent; 1 , minimal; 2 , mild; 3 , moderate; 4 , marked) in the stomahc mucosa of mice treated N-methyl-N-nitrosourea (MNU), Helicobacter felis and/or H. pylori. Data are presented as means \pm SEMs. $* P<0.05$ compared with controls at the same time-point. 
A

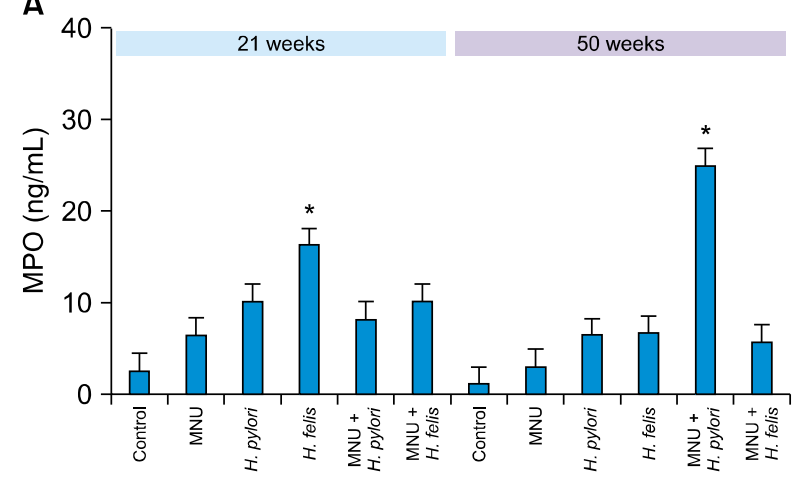

B
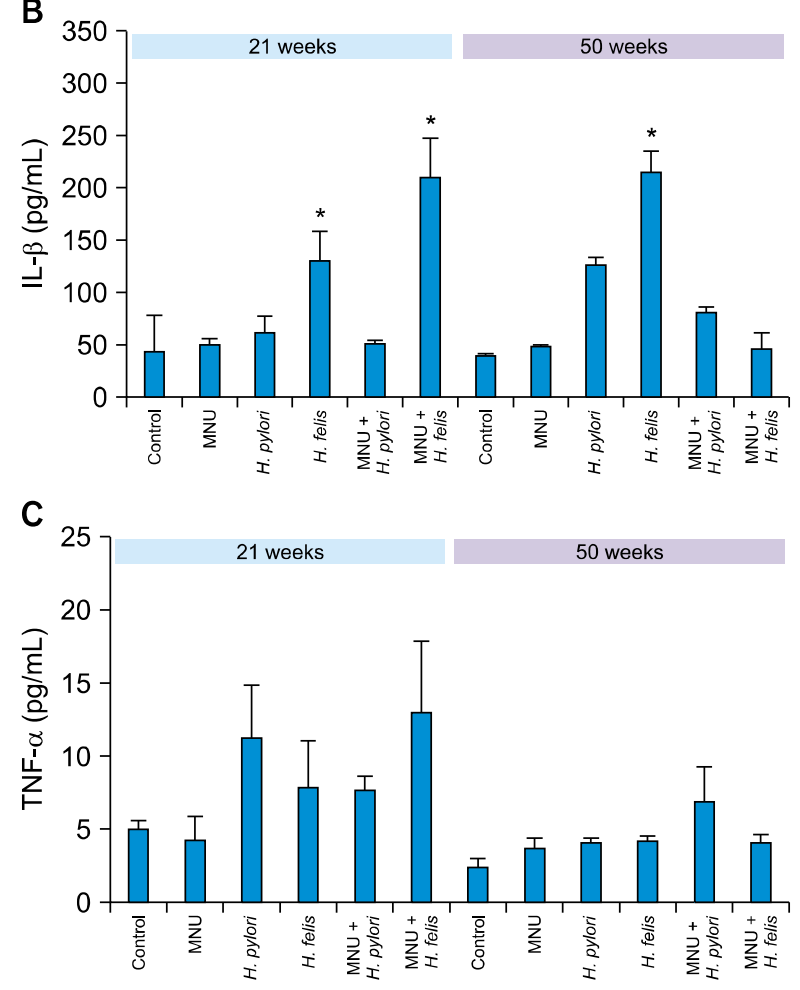

Figure 3. The levels of gastric mucosal myeloperoxidase, interleukin- $1 \beta$, and TNF- $\alpha$ at 21 and 50 weeks. Scraped mucosa (10 mg) was homogenized and the cell suspensions were centrifuged. (A) Myeloperoxidase (MPO), (B) TNF- $\alpha$, and (C) interleukin (IL)-1 $\beta$ levels in the supernatants were measured with ELISA kit following the manufacturer's instructions. Data are presented as means \pm SEMs. MNU, N-methyl-N-nitrosourea. $* P<0.05$ compared with control mice at the same time-point.

mice $(P<0.05)$ (Fig. 3B). The gastric mucosal TNF- $\alpha$ did not show any statistical significance compared with control mice and there was no correlation between the number of weeks and gastric mucosal TNF- $\alpha$ level (Fig. 3C).

\section{DISCUSSION}

In this study, we observed that the incidence of gastric tumors, including adenomas and adenocarcinomas, was higher in the groups of mice infected with $H$. felis with or without MNU. $H$. pylori SS1 alone could cause chronic gastritis with hyperproliferation of the gastric mucosa; however, it did not cause gastric cancer at 50 weeks. In contrast, $H$. felis provoked more severe inflammation, glandular atrophy, metaplasia, dysplastic change, and with MNU, $H$. felis caused gastric cancer at 50 weeks. In addition, we analyzed the levels of gastric mucosal pro-inflammatory cytokines such as IL- $1 \beta$ and TNF- $\alpha$.

MNU-induced tumors in mice are located mainly in the gastric antrum, and pathologically are uniformly well differentiated or moderately differentiated adenocarcinomas. ${ }^{8}$ MNU treatment (0.5 mg, weekly, intragastric intubation) in $\mathrm{BALB} / \mathrm{c}$ mice after removal of the forestomach induced well-differentiated adenocarcinoma in the glandular stomach of mice with $100 \%$ incidence within 40 weeks. ${ }^{3}$ The efficiency of tumor induction by MNU was found to depend on its concentration rather than total intake, ${ }^{12}$ and MNU administered in drinking water at 240 ppm in alternate weeks (total exposure; 5 weeks) was effective in inducing gastric cancer in six strains of mice that were studied. ${ }^{13}$

However, the MNU model of gastric carcinogenesis does not proceed through a classical atrophy-metaplasia-dysplasia sequence and this is a drawback of the MNU mouse model. Several studies showed that pretreatment with MNU prior to Helicobacter infection caused more severe pre-neoplastic changes and increased the occurrence of gastric cancer. Han et al. ${ }^{11}$ found that $>80 \%$ of experimental C57BL/ 6 mice exhibited adenoma or adenocarcinoma in their glandular stomach in 12 months after treatment of $\mathrm{MNU}+H$. pylori SS1, suggesting that $H$. pylori infection clearly enhanced the development of gastric carcinogenesis. Tomita et al. ${ }^{10}$ demonstrated that nearly $100 \%$ of experimental mice presented with pre-neoplastic lesions or adenocarcinoma following 36 weeks of treatment in the MNU + $H$. felis group. It is also reported that $\mathrm{MNU}+$ Helicobacter spp. infection induced a high proportion of gastric cancer than those of MNU only in Mongolian gerbils. ${ }^{14,15}$ Therefore Helicobacter spp. infection followed by MNU treatment thought similar to the pathogenesis of human gastric cancer. ${ }^{8}$

In this study, the incidence of gastric adenoma or adenocarcinoma was higher in the $H$. felis-infected mice group with or without MNU compared with those of the control group or $H$. pylori-infected group. While a chronic colonization of the mouse stomach by H. felis and H. pylorisS1 leads to chronic gastritis and 
premalignant lesions, there is an important difference between the two. Almost all H. felis infections in the C57BL/6 mice develop into gastric adenocarcinoma via sequential steps from intestinal metaplasia and dysplasia, ${ }^{6,7}$ which is very similar to the human gastric carcinogenesis. In contrast, H. pylori SS1 failed to induce gastric adenocarcinoma in most mouse species. Kim et al. ${ }^{16}$ observed H. pylori SS1-infected C57BL/6 mice for 80 weeks and reported that while hyperplastic gastritis or chronic atrophic gastritis was easily induced, no incidence of dysplasia or gastric adenocarcinoma was observed, and explained the reason to be the balance between cell proliferation and apoptosis.

Compared to previous studies, the incidence of gastric tumors was very low and this is a limitation of present study. We believe that this discrepancy arises as a result of two causes. Firstly, we used a relatively low concentration of MNU (200 ppm) compared with that used in other studies. Han et al. ${ }^{11}$ demonstrated an increased gastric tumor rate by $\mathrm{MNU}$ in a dose-dependent manner and $240 \mathrm{ppm}$ displayed the lowest tumor incidence rate. Secondly, we inoculated Helicobacter species five times every alternate day rather than three times every alternate day, which was used in other studies. According to our previous experimental results, inoculation five times every alternate day increased Helicobacter density and therefore we used this protocol. ${ }^{9}$ However, it is not clear whether five times inoculation increases gastric inflammation.

In conclusion, administration of MNU before $H$. felisinfection provokes severe inflammation, and eventually induces gastric cancer. However, it seems that the effect of MNU in $H$. pylori SS1-induced gastric carcinogenesis is lower than that of $H$. felis. IL-1 $\beta$ plays an important role in $H$. felis gastric inflammation; however, TNF- $\alpha$ is not involved in the inflammatory process.

\section{ACKNOWLEDGMENTS}

This work was supported by a National Research Foundation (NRF) of Korea grant for the Global Core Research Center (GCRC) funded by the Korea government (MSIP) (No. 2011-0030001).

\section{CONFLICTS OF INTEREST}

No potential conflicts of interest were disclosed.

\section{REFERENCES}

1. Lee JY, Kim N, Nam RH, Choi YJ, Seo JH, Lee HS, et al. No correlation of inflammation with colonization of Helicobacter pylori in the stomach of mice fed high-salt diet. J Cancer Prev 2014;19:144-51.

2. Lee JY, Kim N, Choi YJ, Nam RH, Lee S, Ham MH, et al. Anti-inflammatory and anti-tumorigenic effects of Açai berry in Helicobacter felis-infected mice. J Cancer Prev 2016;21:48-54.

3. Tatematsu M, Ogawa K, Hoshiya T, Shichino Y, Kato T, Imaida K, et al. Induction of adenocarcinomas in the glandular stomach of BALB/C mice treated with N-methyl-N-nitrosourea. Jpn J Cancer Res 1992;83:915-8.

4. Li Q, Jia Z, Wang L, Kong X, Li Q, Guo K, et al. Disruption of Klf4 in villin-positive gastric progenitor cells promotes formation and progression of tumors of the antrum in mice. Gastroenterology 2012;142:531-42.

5. Tsukamoto T, Mizoshita T, Tatematsu M. Animal models of stomach carcinogenesis. Toxicol Pathol 2007;35:636-48.

6. Rogers AB, Fox JG. Inflammation and cancer. I. Rodent models of infectious gastrointestinal and liver cancer. Am J Physiol Gastrointest Liver Physiol 2004:286:G361-6.

7. Cai X, Carlson J, Stoicov C, Li H, Wang TC, Houghton J. Helicobacter felis eradication restores normal architecture and inhibits gastric cancer progression in C57BL/6 mice. Gastroenterology 2005:128:1937-52

8. Hayakawa Y, Fox JG, Gonda T, Worthley DL, Muthupalani S, Wang TC. Mouse models of gastric cancer. Cancers (Basel) 2013;5:92-130.

9. Lee JY, Kim N, Choi YJ, Nam RH, Choi YJ, Kwon YH, et al. Histologic findings and inflammatory reactions after long-term colonization of Helicobacter felis in C57BL/6 mice. J Cancer Prev 2014:19:224-30.

10. Tomita H, Takaishi S, Menheniott TR, Yang X, Shibata W, Jin G, et al. Inhibition of gastric carcinogenesis by the hormone gastrin is mediated by suppression of TFF 1 epigenetic silencing. Gastroenterology 2011;140:879-91.

11. Han SU, Kim YB, Joo HJ, Hahm KB, Lee WH, Cho YK, et al. Helicobacter pylori infection promotes gastric carcinogenesis in a mice model. J Gastroenterol Hepatol 2002;17:253-61.

12. Yamachika $T$, Nakanishi $H$, Inada $K$, Tsukamoto $T$, Shimizu N, Kobayashi K, et al. N-methyl-N-nitrosourea concentration-dependent, rather than total intake-dependent, induction of adenocarcinomas in the glandular stomach of BALB/C mice. Jpn J Cancer Res 1998;89:385-91.

13. Yamamoto M, Furihata C, Ogiu T, Tsukamoto T, Inada Ki, Hirano $\mathrm{K}$, et al. Independent variation in susceptibilities of six different mouse strains to induction of pepsinogen-altered pyloric glands and gastric tumor intestinalization by N-methyl-N-nitrosourea. Cancer Lett 2002;179:121-32.

14. Maruta F, Sugiyama A, Ishida K, Ikeno T, Murakami M, Kawasaki $\mathrm{S}$, et al. Timing of N-methyl-N-nitrosourea administration affects gastric carcinogenesis in Mongolian gerbils infected with Helicobacter pylori. Cancer Lett 2000;160:99-105.

15. Cao X, Tsukamoto T, Nozaki K, Tanaka H, Shimizu N, Kaminishi $\mathrm{M}$, et al. Earlier Helicobacter pylori infection increases the risk for the N-methyl-N-nitrosourea-induced stomach carcinogenesis in Mongolian gerbils. Jpn J Cancer Res 2002;93:1293-8.

16. Kim DH, Kim SW, Song YJ, Oh TY, Han SU, Kim YB, et al. Long-term evaluation of mice model infected with Helicobacter pylori: focus on gastric pathology including gastric cancer. Aliment Pharmacol Ther 2003;18 Suppl 1:14-23. 